\title{
Internal resonances in periodically modulated long Josephson junctions
}

\author{
Larsen, Britt Hvolbæk; Mygind, Jesper; Ustinov, Alexey V.
}

Published in:

I E E E Transactions on Applied Superconductivity

Link to article, DOI:

$10.1109 / 77.403210$

Publication date:

1995

Document Version

Publisher's PDF, also known as Version of record

Link back to DTU Orbit

Citation (APA):

Larsen, B. H., Mygind, J., \& Ustinov, A. V. (1995). Internal resonances in periodically modulated long Josephson junctions. I E E E Transactions on Applied Superconductivity, 5(2), 2947-2950.

https://doi.org/10.1109/77.403210

\section{General rights}

Copyright and moral rights for the publications made accessible in the public portal are retained by the authors and/or other copyright owners and it is a condition of accessing publications that users recognise and abide by the legal requirements associated with these rights.

- Users may download and print one copy of any publication from the public portal for the purpose of private study or research.

- You may not further distribute the material or use it for any profit-making activity or commercial gain

- You may freely distribute the URL identifying the publication in the public portal

If you believe that this document breaches copyright please contact us providing details, and we will remove access to the work immediately and investigate your claim. 


\title{
Internal resonances in periodically modulated long Josephson junctions
}

\author{
Britt H. Larsen, Jesper Mygind and Alexey V. Ustinov \\ Physics Department, Technical University of Denmark, 2800 Lyngby, Denmark
}

\begin{abstract}
Current-voltage $(I-V)$ characteristics of long Josephson junctions with a periodic lattice of localized inhomogeneities are studied. The interaction between the moving fluxons and the inhomogeneities causes resonant steps in the IV-curve. Some of these steps are due to a synchronization to resonant Fiske modes in the sub-junctions formed between the inhomogeities. The voltage positions of the resonant steps oscillate as function of the applied magnetic field with a period corresponding to the inclusion of one magnetic flux quantum, $\Phi_{0}=h / 2 e$, per sub-junction. A qualitative explanation that takes into account the interaction between the resonance in the sub-junction and the magnetic flux density waves excited in the whole junction is given.
\end{abstract}

\section{INTRODUCTION}

The motion of magnetic flux quanta in long Josephson junctions (i. e. with one dimension much larger than the Josephson penetration depth, $\lambda_{J}$ ) has attracted much interest over the last decades. If a periodic modulation (a lattice of inhomogeneities) is made in the long junction a number of new effects occur [1]. The commensurability between the inhomogeneities and the chain of fluxons in the junction will affect not only the static [2] but also the dynamic behavior of the junction [3],[4]. Also in 2-D arrays of Josephson junctions the fluxon dynamics is influenced by commensurability as seen in the measurements of fractional giant Shapiro steps [5].

One motivation for making a long junction with inhomogeneities was to search for an oscillator better than the highly damped homogeneous long Josephson junction (the so-called flux flow oscillator). It is possible that higher power or better spectral purity in specific frequency ranges can be obtained when synchronized oscillations take place in the sub-junctions formed by the lattice of equidistantly spaced inhomogeneities. On the other side, long junctions with periodic inhomogenieties have some similarities to the parallel 1-D array of short junctions which form the basic transmission line in Rapid Single Flux Quantum (RSFQ) electronics.

A Josephson junction is said to be homogeneous if the

B. H. Larsen, e-mail bhl@mips.fys.dtu.dk, fax +45 4593 1669; J. Mygind, e-mail myg@mips.fys.dtu.dk; A. V. Ustinov, Institute for Thin Films and Ion Technology, Research Center (KFA), D52425 Jülich, Germany. e-mail a.ustinov@kfa-juelich.de, fax +49 2461612940. local junction parameters are independent of the spatial coordinates. An inhomogeneity is defined as a localized region where the parameters of the tunneling barrier are significantly different from the rest of the junction. In the following we consider a long 1-D tunnel junction where the size of each of the $n$ inhomogeneites in the $x$-direction is $r_{0}$ and the lattice period is $a$. The inhomogeneities change the equation of motion for the phase difference, $\phi(x, t)$, between the electrodes. In general, the spatial variation of all the coefficients in the perturbed sine-Gordon equation as well as the finite width of the inhomogeneities must be included. In most numerical and analytical treatments of the system [1],[4] it has been assumed that the inhomogeneities only cause a change in the critical current density and that the size of the inhomogeneities are much smaller than $\lambda_{J}$. In this case, the inhomogenities can be modelled by $\delta$-functions in the critical current density.

For a junction with a periodic lattice of inhomogeneities the magnetic diffraction pattern, $I_{c}(B)$, displays a number of equidistant maxima. $B$ is the applied magnetic field. The critical current $I_{c}$ is proportional to the total pinning force on the fluxon chain, and since this pinning force has a maximum when all the inhomogeneities contribute equally, these maxima arise whenever there is an integer number of fluxons per inhomogeneity. The fields where these maxima occur are called the commensurability fields, $B_{p}=p \cdot B_{1}$, where the period $B_{1}$ corresponds to one additional flux quantum per inhomogeneity.

In the vicinity of the commensurability fields the fluxon chain is strongly modulated by the lattice of inhomogeneities. The resulting configuration can be viewed as a chain of fluxons with a defect. This density modulation or defect acts as a soliton itself and is called a supersoliton [4]. One or several of these supersolitons can perform a flux flow type motion and create a new resonance in the junction. The voltage position of the supersoliton step depends on the number of supersolitons in the junction. The difference between the applied magnetic field and the nearby value of the commensurability field, $B_{p}$, is proportional to the number of supersolitons in the junction:

$$
n_{\mathrm{ss}}=\frac{L \Lambda}{\Phi_{0}}\left(B-B_{p}\right),
$$

where $\Lambda=2 \lambda_{L}+t$ is the magnetic thickness of the barrier, i. e. the sum of the London penetration depths, $\lambda_{L}$, of the two electrodes and the barrier thickness, $t$. If the number of supersolitons is negative it corresponds to missing fluxons (anti-supersolitons) in the chain whereas a positive 
TABLE I

JUNCTION PARAMETERS.

\begin{tabular}{lcc}
\hline sample & $\mathrm{C}-2$ & $\mathrm{D}-2$ \\
\hline number of inhomogeneities,n & 5 & 9 \\
length, $\mathrm{L}(\mu \mathrm{m})$ & $480 \pm 0.5$ & $480 \pm 0.1$ \\
width, W $(\mu \mathrm{m})$ & $10 \pm 0.5$ & $10 \pm 0.5$ \\
$\mathrm{r}_{0}(\mu \mathrm{m})$ & $10 \pm 0.5$ & $10 \pm 0.5$ \\
a $(\mu \mathrm{m})$ & $98 \pm 0.5$ & $49 \pm 0.5$ \\
a/ro & $9.8 \pm 0.5$ & $4.9 \pm 0.1$ \\
$I_{c}(\mathrm{~mA})$ & $2.6 \pm 0.1$ & $2.2 \pm 0.1$ \\
$j_{c}=I_{c} /\left[W \cdot\left(L-n r_{0}\right)\right]\left(10^{6} \mathrm{~A} / \mathrm{m}^{2}\right)$ & $0.60 \pm 0.02$ & $0.56 \pm 0.02$ \\
$\lambda_{J}(\mu \mathrm{m})$ & $75 \pm 2$ & $78 \pm 2$ \\
$\mathrm{~L} / \lambda_{J}$ & $6.4 \pm 0.5$ & $6.2 \pm 0.5$ \\
$\bar{c} / c_{0}$ & $0.031 \pm 0.002$ & $0.031 \pm 0.002$ \\
$I_{1}(\mathrm{~mA})$ & $2.2 \pm 0.1$ & $4.2 \pm 0.1$ \\
$I_{c} 1(\mathrm{~mA})$ & $1.0 \pm 0.1$ & $1.0 \pm 0.1$ \\
\hline
\end{tabular}

$c_{0}$ is the light velocity in vacuum.

number of supersolitons corresponds to extra fluxons in the junction as compared to the commensurability configuration. The asymptotic voltage of the supersoliton step depend on the external magnetic field:

$$
V_{\mathrm{sss}}= \pm \bar{c} \Lambda\left(B-B_{p}\right),
$$

where $+(-)$ refers to supersolitons (anti-supersolitons) and $\bar{c}$ is the Swihart velocity.

The long Josephson junction with a lattice of regularly spaced inhomogeneities may also be considered a system of parallel-coupled shorter Josephson junctions (subjunctions) [3]. For certain voltages, the velocity of the fluxons is synchronized with a Fiske mode of the subjunctions. The Fiske mode is excited by the radiation emitted when the fluxons pass the inhomogeneities. Due to the coherence between oscillations excited in neighboring sub-junctions the current step from such a resonance is called a super-radiation step [3]. The voltage positions of the super-radiation steps coincide with the Fiske steps of the sub-junctions:

$$
V_{\mathrm{srs}}=n \Phi_{0} \frac{\bar{c}}{2 a^{\prime}}
$$

where $a^{\prime}$ is the effective length of the sub-junctions, expected to be $a-r_{0}$.

So far the resonances induced in long periodicallymodulated junctions have been considered from two different models discussed above, as being due either to supersolitons or to super-radiation. In this paper we suggest an approach which links together these two alternative points of view. We propose a rather simple qualitative picture which accounts for main features of the fluxon dynamics.

\section{SAMPLES}

The junctions were fabricated [3] as a $\mathrm{Nb}-\mathrm{NbO}_{x}-\mathrm{Pb}$ structure where the inhomogeneities are created by a local increase of the oxide barrier using an additional insulating layer made of $\mathrm{SiO}$. The thickness of $100 \mathrm{~nm}$ was sufficient
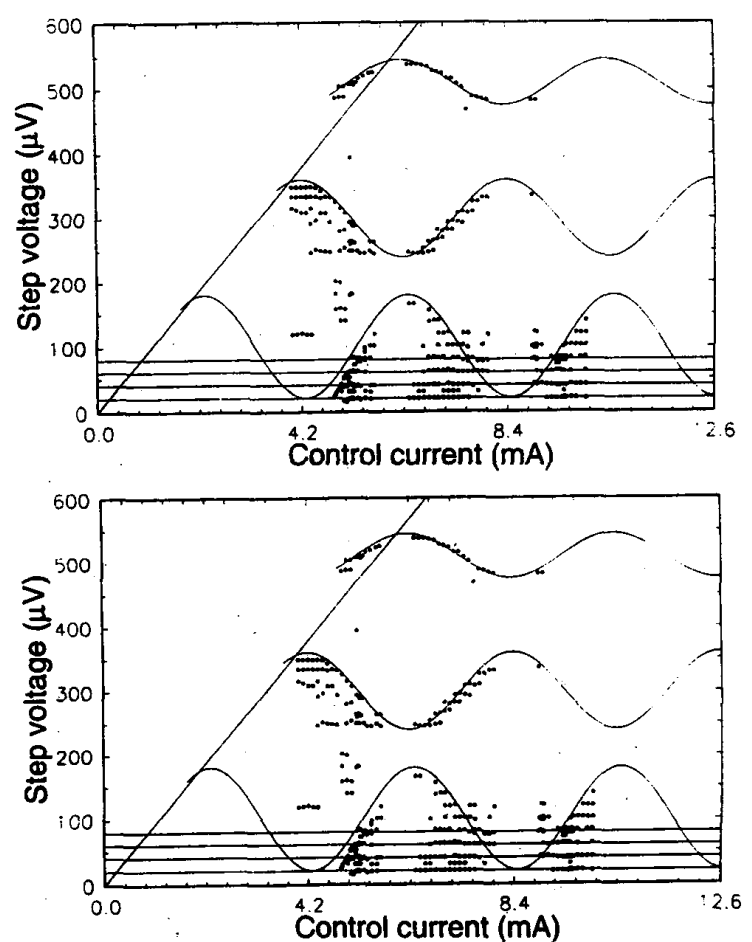

Fig. 1. The maximum step voltage as a function of the applied magnetic field (proportional to the control current $I_{m}$ ) for (a) junction C-2 and (b) junction D-2.

to prevent any tunneling. This, however, does not destroy the long junction properties because the inhomogeneities are shorter than $\lambda_{J}$, and thus permit the fluxons to pass.

The external magnetic field is applied to the junctions by feeding a dc current through one of the electrodes (in the $x$-direction). This control current, $I_{m}$, generates a proportional magnetic field in the plane of the barrier predominantly perpendicular to the $x$-direction.

\section{EXPERIMENTAL RESULTS}

For an applied magnetic field larger than the first critical field $B_{c 1}$ the $I V$-characteristic of the junction exhibits a large number of different current steps. In the following the voltage position, $V_{s}$, of the step refers to the voltage at the largest attainable step current.

Fig. 1 shows the step voltage versus applied magnetic field for two different junctions. If a step is independent of magnetic field, it gives a horizontal line in the $\left(V_{s}, I_{m}\right)$ diagram whereas, if $V$, is proportional to the applied magnetic field it gives a line with a slope equal to the proportionality constant.

Junction C-2 (Fig. 1a) has five inhomogeneities (Table I). The equidistant steps at low voltage are Fiske steps, and one gets $\Delta V_{\mathrm{fs}}=(20 \pm 1) \mu \mathrm{V}$. All observed steps are at lower voltage than the flux flow step, i. e. below the straight line in Fig. 1(a) corresponding to the voltage, 


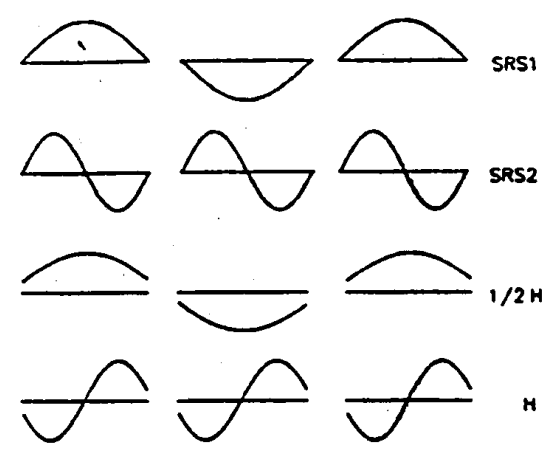

Fig. 2. The variation of current density for super-radiation step No. 1 , and 2 and for magnetic fields $\frac{1}{2} B_{1}$, and $B_{1}$

$V_{\mathrm{ff}}$, of the flux flow step. The measured slope of this line, $d V_{\mathrm{ft}} / d I_{m}=(84 \pm 4) \mu \mathrm{V} / \mathrm{mA}$, coincides with the value predicted by the model, $d V_{\mathrm{ff}} / d I_{m}=\bar{c} \Phi_{0} / I_{c 1} \pi \lambda_{J}=(81 \pm$ 9) $\mu \mathrm{V} / \mathrm{mA}$.

A number of almost equidistant steps with voltage positions oscillating around a fixed voltage as a function of the magnetic field are seen. These are the super-radiation steps. According to the model [3], they are due to excitations of Fiske modes in the sub-junctions formed between successive inhomogeneities. Comparing the difference between the average positions of super-radiation steps with the Fiske steps one gets: $V_{\mathrm{Brs}}=(92 \pm 5) \mu \mathrm{V}$, $V_{\mathrm{srd}} / V_{\mathrm{fs}}=4.6 \pm 0.4$. The ratio between the voltages of the super-radiation step and the Fiske step should give the ratio between the total length of the junction and the length of the sub-junctions where the resonances originate, $L /\left(a-r_{0}\right)=5.5 \pm 0.6$. From this ratio between the voltages it can be seen that the effective length of each sub-junction probably is longer that the distance between two inhomogeneities. It is likely that the standing wave pattern in each of the sub-junctions extends into the inhomogeneities.

The junction D-2 has nine inhomogeneities. The low voltage steps which are nearly independent of the magnetic field are the Fiske steps of the whole long junction: $\Delta V_{\mathrm{fs}}=(20 \pm 1) \mu \mathrm{V}$. Fig. 1(b) shows measurements of voltage steps for control currents $I_{m}$ between $I_{1}$ and $I_{2}$ (corresponding to the commensurability fields $B_{1}$ and $B_{2}$, respectively). The first supersoliton step is seen as an individual step for small voltages, i. e. close to $I_{1}$, but it is also seen locked to Fiske steps for larger voltages. Two super-radiation steps are seen. They are oscillating with a period approximately equal to $I_{1}$ and they are out of phase. The difference between their average voltages is $V_{\mathrm{srs}}=(200 \pm 20) \mu \mathrm{V}$ giving $V_{\mathrm{srs}} / V_{\mathrm{fs}}=10 \pm 1$. This should be compared to $L /\left(a-r_{0}\right)=12 \pm 1$, and again it seems that the effective length of the sub-junctions giving the super-radiation steps is slightly longer than the physical distance between the inhomogeneities. The slope of $V_{\mathrm{Bss}}\left(I_{m}\right)$ is measured to $(90 \pm 10) \mu \mathrm{V} / \mathrm{mA}$ in agreement with the value predicted by theory: $d V_{\mathrm{sss}} / d I_{m}=\bar{c} \Phi_{0} /$
$I_{\mathrm{c} 1} \pi \lambda_{J}=(78 \pm 9) \mu \mathrm{V} / \mathrm{mA}$. Summarizing the results, it was found that the slope of the dependence of the flux flow step on the control current agrees with the calculated value for both junctions. Furthermore, the ratio between the average distance between the superradiation steps and the Fiske step of the long junction agrees with the ratio between the length of the long junction and the distance between the inhomogeneities. In both junctions $L /\left(a-r_{0}\right)$ is greater than $V_{\mathrm{srg}} / V_{\mathrm{fs}}$, suggesting that the resonant Fiske mode in the sub-junctions giving the superradiation steps, extends slightly into the inhomogeneities.

\section{MODEL}

From the simple model where the super-radiation steps are predicted to be at voltages corresponding to the Fiske steps of the sub-junction, their voltage positions are expected to be independent of the applied magnetic field. Instead, we observe that the voltage positions of the super-radiation steps seem to follow an almost sinusoidal oscillation. The period is approximately $I_{1}$ and neighboring super-radiation steps oscillate out of phase. A similar behavior has previously been observed [3].

A possible explanation of the oscillation of the superradiation steps can be given looking at the currunt den$\operatorname{sity} \sin (\phi)$ along the junction. The resonance giving the super-radiation steps corresponds to a Fiske step in each sub-junction. In Fig. 2 the current density along a long junction consisting of three sub-junctions (i. e. with 2 inhomogeneities) is shown for the first two super-radiation steps. The current distribution is also shown for evenly distributed fluxons at magnetic fields equal to $\frac{1}{2} B_{1}$ and $B_{1}$. It is seen that the current density distribution in the junction at the first super-radiation step is similar to the current density distribution at $\frac{1}{2} B_{1}$; for the second superradiation step the current density distribution is similar to that at $B_{1}$, etc. Therefore at $\frac{1}{2} B_{1}$ the resonance giving the first super-radiation step is likely to be excited i. e. the first super-radiation step will have maximum amplitude. The second super-radiation step will have maximum amplitude at $B_{1}$. This explains the voltage positions of the super-radiation steps when they coincide with the flux flow step.

Below, we qualitatively discuss the mechanism for the excitation of super-radiation steps at higher magnetic fields. The second super-radiation step is used as an example. At $B=B_{2}$ the voltage position of the second super-radiation step is the same as at $B_{1}$ (point $A$ in Fig. 3). This can be explained if the dynamical state is a superposition of two states: Let one fluxon be pinned for each inhomogeneity similar to the stationary state at $B_{1}$. This takes up half of the available fluxons in the junction. The other half of the fluxons moves in a chain and synchronizes the Fiske steps of the sub-junctions similar to the dynamical state of the second super-radiation 


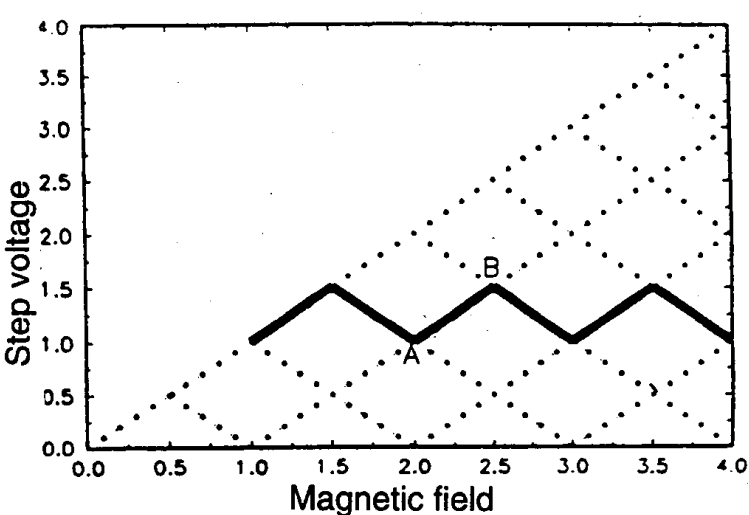

Fig. 3. The model's prediction of the voltage step position as function of the magnetic field for the 2 nd super-radiation step.

step at $B_{1}$. The pinned fluxons do not contribute to the voltage over the junction so the total voltage of the second super-radiation step at $B_{2}$ is identical to the voltage at $B_{1}$. By generalizing to higher commensurate states and higher Fiske modes in the sub-junctions (i.e. higher super-radiation steps), one finds that at fields $n B_{1}$ the even super-radiation steps are at the same voltage positions as they were on the flux flow line. In a similar way, at fields $\left(m+\frac{1}{2}\right) B_{1}$ the odd super-radiation steps are at the same voltage positions as they were at the flux flow line.

For fields between $\frac{3}{2} B_{1}$ and $\frac{5}{2} B_{1}$ the voltage position of the second super-radiation step can be explained as follows. First, let the field decrease from $B_{2}$ to $\frac{3}{2} B_{1}$ and assume that the fluxon motion synchronizing the Fiske modes in the sub-junctions is unchanged. Then the decrease in the field only influences the commensurate state. The decrease in field gives a defect in the commensurate state with properties of an antifluxon. This antisuperfluxon moves in the opposite direction of an ordinary fluxon and therefore the voltage from this state rises linearly with decreasing magnetic field (with a slope equal to that of the flux flow), and the total voltage over the junction will increase when the magnetic field is decreased from $B_{2}$ to $\frac{3}{2} B_{1}$.

When increasing the field from $B_{2}$ to $\frac{5}{2} B_{1}$, it is again assumed that the synchronized motion is undisturbed and that the extra flux is introduced as a defect or superfluxon in the commensurate state. In this case the voltage over the junction will increase for increasing magnetic field. At $\frac{5}{2} B_{1}$ (point B in Fig. 3) there is one superfluxon for every second inhomogeneity. This fluxon configuration is identical to the third commensurate state with one antisuperfluxon for every second inhomogeneity. Increasing the magnetic field above $\frac{5}{2} B_{1}$, it is favorable that the commensurate state has one extra fluxon per inhomogeneity and some anti-supersolitons instead of supersolitons. Thus, the voltage position of the super-radiation step decreases for increasing magnetic field. The branches from $2 B_{1}$ and $3 B_{1}$ of the second super-radiation step will then join in point $B$. These arguments can be generalized to the $n$th superradiation step independently of whether $\mathbf{n}$ is even or odd.

This proposed model explains the oscillations of the voltage position of the super-radiation steps, why the period is $B_{1}$, and why neighboring super-radiation steps oscillate out of phase. The only property of the oscillation of the superradiation steps that is not accounted for is the fact that the oscillations are smoothed to become almost sinusoidal. According to the above argument the oscillations should be triangular.

\section{CONCLUSION}

We have measured the voltage steps of a long Josephson junction with a lattice of equidistant inhomogeneities as a function of applied magnetic field. The simple model describing the super-radiation steps as Fiske steps in the sub-junctions formed between inhomogeneities does not account for the oscillations in the voltage position when the magnetic field is changed. We propose a qualitative model that explains this oscillations. This model takes into account the motion of the defects in the fluxon chain (supersoliton) at magnetic fields where the chain of fluxons is incommensurate with the lattice of inhomogeneities.

\section{ACKNOWLEDgMENT}

We thank V. A. Oboznov for making the samples. The hospitality of the Danish Institute of Fundamental Metrology (DFM) where the measurements were performed is gratefully acknowledged. One of the authors (AVU) acknowledges the support from the Danish Research Academy.

\section{REFERENCES}

[1] A. V. Ustinov,"Solitons in long Josephson junctions with inhomogeneities", in: Nonlinear Superconductive Electronics and Josephson Devices, eds. G. Costabile et al. (Plenum Press, New York, 1991) 315.

[2] B. H. Larsen, J. Mygind and A. V. Ustinov, "Commensurability between fluxons and inhomogeneities in a long Josephson junction", Phys. Lett. A 193, 359 (1994).

[3] B. A. Malomed and A. V. Ustinov, "Super-radiant multifluxon dynamics in a system of parallel-coupled Josephson junctions",Phys. Rev. B 41, 254 (1990).

[4] B. A. Malomed and V. A. Oboznov and A. V. Ustinov, "'Supersolitons' in periodically inhomogeneous long Josephson junctions", Zh. Eksp. Teor. Fiz. 97 (1990) 924 [Sov. Phys. JETP 70 (1990) 518].

[5] S. P. Benz, M. S. Rzchowski, M. Tinkham, and C. J. Lobb, "Fractional Giant Shapiro Steps and Spatially Correlated Phase Motion in 2D Josephson Array", Phys. Rev. Lett. 64, 693 (1990).

[6] V. P. Koshelets, A. V. Shchukin, S. V. Sintov, and L. V. Filippenko, "Superconducting millimeter wave oscillators and SIS mixers integrated on a chip",IEEE Trans. Appl. Supercond. 3, 2524 (1993). 